\title{
People Tracking with Human Motion Predictions from Social Forces
}

Matthias Luber

Johannes A. Stork

Abstract-For many tasks in populated environments, robots need to keep track of current and future motion states of people. Most approaches to people tracking make weak assumptions on human motion such as constant velocity or acceleration. But even over a short period, human behavior is more complex and influenced by factors such as the intended goal, other people, objects in the environment, and social rules. This motivates the use of more sophisticated motion models for people tracking especially since humans frequently undergo lengthy occlusion events.

In this paper, we consider computational models developed in the cognitive and social science communities that describe individual and collective pedestrian dynamics for tasks such as crowd behavior analysis. In particular, we integrate a model based on a social force concept into a multi-hypothesis target tracker. We show how the refined motion predictions translate into more informed probability distributions over hypotheses and finally into a more robust tracking behavior and better occlusion handling. In experiments in indoor and outdoor environments with data from a laser range finder, the social force model leads to more accurate tracking with up to two times fewer data association errors.

\section{INTRODUCTION}

People tracking is a key technology for mobile robots to be safely and efficiently deployed in populated environments. Most related work in people tracking [1], [2], [3], [4] make weak assumptions on the motion of humans and employ either the Brownian model or the constant velocity motion model. The former makes no assumptions about the target dynamics, the latter assumes linear target motion and constant velocity. Both models predict future states merely based on the history of past states. However, human motion follows more complex, non-linear patterns. People are usually driven by an inner motivation towards some goal, they are influenced by obstacles and other people along their path, and follow social rules. In other words, human motion is influenced by the physical and social constraints of the environment.

Better motion models for people tracking have been proposed. In [5], the robot first learns goal locations in the environment from people trajectories. Human motion is then predicted along paths computed by a planner from the actual location of the person being tracked to the estimated goal location. Liao et al. [6] extract a Voronoi graph from a map of the environment and

All authors are with the Social Robotics Lab, Department of Computer Science, University of Freiburg, Germany \{luber,stork,tipaldi,arras\}@informatik.uni-freiburg.de.

\author{
Gian Diego Tipaldi Kai O. Arras
}

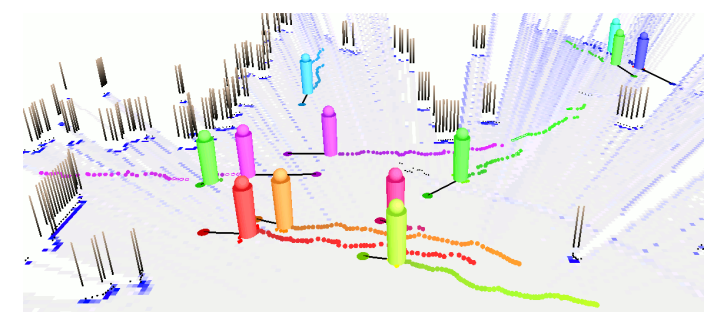

Fig. 1. Twelve of 150 tracks from the outdoor experiment. The cylinders show the estimated positions of the pedestrians, the colored dots illustrate their past trajectories. The circles connected with lines are the virtual goals. The occupancy grid map is overlaid where darker blue cells indicate higher occupancy probability. Vertical lines visualize static objects.

constrain the state of people to lie on edges of that graph. The motion of people is then predicted along those edges following the topological shape of the environment. Bennewitz et al. [7] learns typical motion patterns that people follow in an environment. The approach collects trajectories of people and combines them to motion patterns using EM clustering. From each pattern a HMM is derived that enables a mobile robot to predict the motion of people. In a recent work, Luber et al. [8] learn spatial priors on human motion in an environment using a non-homogeneous spatial Poisson process. The process is learned by observing people, leading to spatial distributions of where people usually walk, appear or disappear. A place-dependent motion model is then derived from the Poisson process using a sample-based approach.

Models for pedestrian dynamics have also been developed and applied in communities such as quantitative sociology or spatial cognition. They are used for crowd simulation, evacuation dynamics, or building design (Fig. 2). Schadschneider et al. present a taxonomy of various models in [9]. A first group of approaches employ fluid-dynamic and gas-kinetic models [10] in which people are considered particles with their motion being described by fluid-dynamic equations. These approaches are typically deterministic and force-based. This is unlike methods based on cellular automata that are discrete, rule-based dynamical models. They discretize space into cells that can be occupied by at most one person. The dynamics is usually described by a set of rules specifying the probability of moving to the neighboring cells. An extension of this approach is the floor field approach [11], where the transition probability of cells are not fixed but vary dynamically. Ali and Shah [12] combine a floor field approach with an evacuation model to improve 
people tracking. However, given their discrete nature, such motion models cannot be readily applied within a probabilistic tracker that requires proper error propagation in the state prediction step.

The social force model proposed by Helbing et al. [13] is a deterministic continuum model in which interactions between pedestrians are described using the concept of social forces or social fields. These forces model different aspects of motion behaviors, such as the motivation of people to reach a goal, the repulsive effect of walls and other people as well as physical constraints.

In this paper we combine a people tracker with a pedestrian dynamics model for the purpose of more realistic human motion predictions. Among the existing methods, we choose the social force model as it is a simple yet powerful approach with justifications from social psychology. Its building block, the social force, is well explained by psychological and social insights. We extend the state-of-the-art of human motion prediction for tracking by two points. First, we use a single outof-the-box model that is able to coherently describe several aspects of pedestrian dynamics such as intention towards a goal, constraints from the environments and from other people. This is in contrast to previous work that uses combinations of methods, such as goal learning and planning [5], Voronoi graph extraction from a map [6] or EM clustering and HMMs [7]. The model requires no learning step. Secondly, our model deals with interpeople relations for motion prediction. During an occlusion event of two persons approaching each other, for instance, the model will not predict their future states into the other person (or into an object). This is an important aspect, as shown in the experiments, that has not been previously considered.

Independently developed from our work, Pellegrini et $a l$. recently proposed the social force model for motion prediction in the context of visual people tracking [14]. In their approach, the person velocity is also accounted for in the energy potential. This is achieved by estimating the closest future distance in the space-time trajectory of targets and use this distance as an additional potential. This enables the system to plan ahead to some extent but comes at the expense of the loss of a closed-form solution. Unlike [14] we consider additional forces from the environment by maintaining a short-term environment model and computing different repulsion forces and physical constraints from static obstacles. We then compare their contribution with those from inter-person influences only.

The paper is structured as follows. Section II introduces the theory of the social force model. Section III describes how the model can be used to compute refined motion predictions. Section IV presents the experimental results followed by the conclusions in section V.

\section{The Social Force Model}

The social force model by Helbing et al. [13], [15] is a computational model in which the interactions between
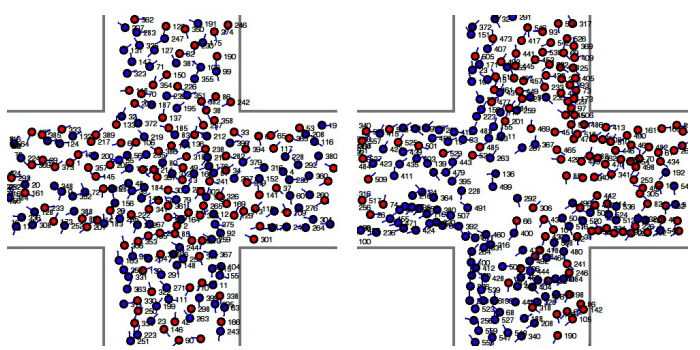

Fig. 2. Example simulation of crowd behavior in a crossing using the social force model. Two groups of pedestrians (shown as red and blue circles) try to traverse the crossing to reach the opposite corridor. Over time, lanes in a twirl-like pattern emerge in a bottom-up fashion as the most efficient motion strategy (left $t=0$, right $t=2 \mathrm{~min}$ ).

pedestrians are described by using the concept of a social force. It is based on the idea that changes in behavior can be explained in terms of social fields or forces. Applied to pedestrians, the social force model accounts for the influence of the environment and other people and describes how the intended direction of motion changes as a function of these influences. The model does not cover cases of multiple options, when people have to actively decide. Game theoretic approaches can be applied in such situations.

Formally, the models assumes that a pedestrian $p_{i}$ with mass $m_{i}$ likes to move with a certain intended velocity $\widehat{\mathrm{v}}_{i}$ in an intended direction $\widehat{\boldsymbol{e}}_{i}$ and therefore tends to adapt his or her velocity $\boldsymbol{v}_{i}$ within a so called relaxation time $\tau_{i}$. This change of velocity is modeled by the personal motivation

$$
\mathbf{F}_{i}^{\text {pers }}=m_{i} \frac{\widehat{\mathrm{v}}_{i} \widehat{\boldsymbol{e}}_{i}-\boldsymbol{v}_{i}}{\tau_{i}} .
$$

The relaxation time is the time interval needed to reach the intended velocity and the intended direction.

In the presence of other people or objects in the environment, a pedestrian might not be able to keep the intended direction and velocity. In the social force model, repulsive effects from these influences are described by an interaction force $\mathbf{F}_{i}^{\text {soc }}$. This force prevents humans from walking along their intended direction and is modeled as a sum of forces either introduced by other individuals $p_{j}$ or by static obstacles denoted by subscript $O$

$$
\mathbf{F}_{i}^{\mathrm{soc}}=\sum_{j \in \mathcal{P} \backslash\{i\}} \mathbf{f}_{i, j}^{\mathrm{soc}}+\sum_{o \in \mathcal{O}} \mathbf{f}_{i, o}^{\mathrm{soc}}
$$

with $\mathcal{P}=\left\{p_{i}\right\}_{i=1}^{N_{p}}$ being the set of all people and $\mathcal{O}$ the static objects of the environment. These forces decrease proportional to the distance of their sources and are modeled as

$$
\mathbf{f}_{i, k}^{\mathrm{soc}}=a_{k} e^{\left(\frac{r_{i, k}-d_{i, k}}{b_{k}}\right)} \boldsymbol{n}_{i, k}
$$

where $k \in \mathcal{P} \cup \mathcal{O}$ is either a person or an object of the environment, $a_{k}$ specifies the magnitude and $b_{k}$ the range of the force. In order to calculate the Euclidean distance between $p_{i}$ and entity $k$, pedestrians and objects are assumed to be of circular shape with radii $r_{i}$ and $r_{k}$, 

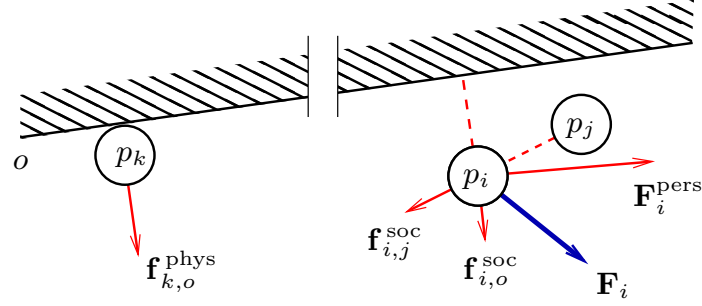

Fig. 3. Forces in the social force model shown as red arrows. Pedestrian $p_{k}$ is affected by the physical force of the static part of the environment. Pedestrian $p_{i}$ is both affected by the wall and an other person $p_{j}$. The superposition of forces exerted to $p_{i}$ is shown as the blue arrow $\mathbf{F}_{i}$.

respectively. Then, distance $d_{i, k}$ is given by the Euclidian distance between the centers, and $r_{i, k}$ is the sum of their radii. The term $\boldsymbol{n}_{i, k}$ is the normalized vector pointing from $k$ to $p_{i}$ which describes the direction of the force.

Given the limited field of view of humans, influences might not be isotropic. This is formally expressed by scaling the forces with an anisotropic factor

$$
\mathbf{f}_{i, k}^{\mathrm{soc}}=a_{k} e^{\left(\frac{r_{i, k}-d_{i, k}}{b_{k}}\right)} \boldsymbol{n}_{i, k}\left(\lambda+(1-\lambda) \frac{1+\cos \left(\varphi_{i, k}\right)}{2}\right)
$$

where $\lambda$ define the strength of the anisotropic factor and

$$
\cos \left(\varphi_{i, k}\right)=-\boldsymbol{n}_{i, k} \cdot \widehat{\boldsymbol{e}}_{i} .
$$

Human motion is not only influenced by personal motivation and reactive behavior towards obstacles or other people but is physically constrained by the environment [15]. Hard constraints restrict the motion and thereby define the walkable area of the environment. Therefore, the social force model introduces a physical force $\mathbf{F}_{i}^{\text {phys }}$ onto pedestrian $p_{i}$ described as

$$
\begin{aligned}
\mathbf{F}_{i}^{\text {phys }} & =\sum_{j \in \mathcal{P} \backslash\{i\}} \mathbf{f}_{i, j}^{\text {phys }}+\sum_{o \in \mathcal{O}} \mathbf{f}_{i, o}^{\text {phys }} \\
\mathbf{f}_{i, k}^{\text {phys }} & =c_{k} g\left(r_{i, k}-d_{i, k}\right) \boldsymbol{n}_{i, k},
\end{aligned}
$$

where $c_{k}$ represents the magnitude of the exerted force. To make the physical forces a real contact force where the circular shapes of $p_{i}$ and $k$ overlap, the function $g$ is defined as $g(x)=x$ if $x \geq 0$ and 0 otherwise.

Finally, human motion is explained by the superposition of all exerted forces. Accordingly, the force $\mathbf{F}_{i}$ changing the motion of individual $p_{i}$

$$
\mathbf{F}_{i}=\mathbf{F}_{i}^{\text {pers }}+\mathbf{F}_{i}^{\mathrm{soc}}+\mathbf{F}_{i}^{\text {phys }} .
$$

Using $\mathbf{F}_{i}$, the basic equation of motion for a pedestrian is then of the general form

$$
\frac{d}{d t} \boldsymbol{v}_{i}=\frac{\mathbf{F}_{i}}{m_{i}}
$$

and describes the movements of $p_{i}$ over time. An illustration of all forces is shown in Fig. 3. The physical force $\mathbf{f}_{k, o}^{\text {phys }}$ that the wall exerts onto person $p_{k}$ is shown. This avoids motion predictions through walls. A superposition of different forces onto pedestrian $p_{i}$ is also shown.

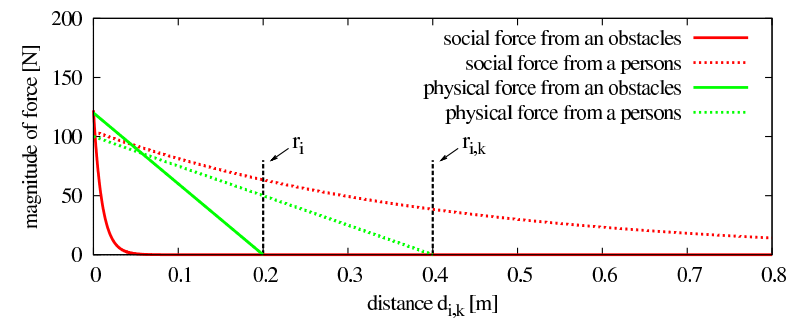

Fig. 4. Typical functions for the exerted forces. The $\mathrm{x}$-axis shows the distance from person $p_{i}$ to an object $o$ or a person $p_{k}$ and the $\mathrm{y}$-axis the magnitude of the forces in Newton. The radius of $p_{i}$ is $r_{i}=0.2 m$ and the sum of the radii of $p_{i}$ and $p_{k}$ is $r_{i, k}=0.4 m$.

The person wants to keep his or her intendend velocity through the motivation $\mathbf{F}_{i}^{\text {pers }}$ but is also influenced by $\mathbf{f}_{i, j}^{\text {soc }}$ from person $p_{j}$ and by $\mathbf{f}_{i, o}^{\text {soc }}$ from the wall. This results in the superimposed force $\mathbf{F}_{i}$ used to adapt the velocity of $p_{i}$.

\section{iII. Motion Prediction Using Social Forces}

Good motion models are particularly important for people tracking as people frequently undergo lengthy occlusion events during interaction with each other or with the environment. In this section, we show how the social force model can be combined with a Kalman filter based tracker to result in a more realistic prediction model of human motion.

Let $\mathbf{x}_{t}=\left(\begin{array}{llll}x_{t} & y_{t} & \dot{x}_{t} & \dot{y}_{t}\end{array}\right)^{T}=\left(\begin{array}{ll}\boldsymbol{x}_{t} & \boldsymbol{v}_{t}\end{array}\right)^{T}$ be the state of pedestrian $p_{i}$ at time $t$ and $\Sigma_{t}$ its $4 \times 4$ covariance matrix estimate. The term $\boldsymbol{x}_{t}$ represents the position and $\boldsymbol{v}_{t}$ the velocity of the pedestrian in Cartesian space. In the remainder we will skip subscript $i$ for the sake of simplicity. The constant velocity motion model is then defined as

$$
p\left(\mathbf{x}_{t} \mid \mathbf{x}_{t-1}\right)=\mathcal{N}\left(\mathbf{x}_{t} ; A \mathbf{x}_{t-1}, A \Sigma_{t-1} A^{T}+Q\right),
$$

with $A$ being the state transition matrix. The entries of $Q$ represent the acceleration capabilities of a human. We extend this model by considering how the state of the pedestrian at a generic time $t$ is influenced by its previous state, other people $\mathcal{P}$ and static obstacles $\mathcal{O}$. We use a discrete time approximation of Eq. 9 within a fixed interval of time $\Delta t$ to obtain $\mathbf{x}_{t}=\xi\left(\mathbf{x}_{t-1}, \mathcal{P}, \mathcal{O}\right)$, where

$$
\xi\left(\mathbf{x}_{t-1}, \mathcal{P}, \mathcal{O}\right)=\left[\begin{array}{c}
\boldsymbol{x}_{t-1}+\boldsymbol{v}_{t-1} \Delta t+\frac{1}{2} \frac{\mathbf{F}}{m} \Delta t^{2} \\
\boldsymbol{v}_{t-1}+\frac{\mathbf{F}}{m} \Delta t
\end{array}\right]
$$

describes how the motion of a pedestrian evolves over time. The change in motion is calculated according to the pedestrian's intended velocity, reactive behavior from interaction forces and physical constraints from the environment, according to Eq. 8. Assuming that the motion is affected by Gaussian noise with zero mean and covariance matrix $Q$ we have

$$
\begin{aligned}
& p\left(\mathbf{x}_{t} \mid \mathbf{x}_{t-1}, \mathcal{P}, \mathcal{O}\right)= \\
& \quad \mathcal{N}\left(\mathbf{x}_{t} ; \xi\left(\mathbf{x}_{t-1}, \mathcal{P}, \mathcal{O}\right), J_{\xi} \Sigma_{t-1} J_{\xi}^{T}+Q\right),
\end{aligned}
$$


where $J_{\xi}=\frac{\partial \xi(\cdot)}{\partial \mathbf{x}}$ is the Jacobian of $\xi(\cdot)$ evaluated at $\mathbf{x}_{t-1}$.

Without external stimuli and deviation from the intended direction and preferred velocity, $\mathbf{F}$ is zero, no social forces are applied to the pedestrian and the social force model falls back onto the constant velocity motion model. The computation of the individual forces is explained in the following sections.

\section{A. Estimating Short-Term Intentions}

The social force model is based on the assumption that each pedestrian has an intended direction of motion and a preferred velocity, both as a result of a higher level goal. However, estimating this goal from the observation of a tracked person involves the problem of intention or activity recognition, an issue beyond the scope of this work. Since we focus on short-term human motion prediction, we make the weak assumption that subjects will continue to pursue his or her short-term intention keeping the current velocity vector.

In doing so, we introduce the concept of virtual goals. A virtual goal is defined as the hypothetical position that a person would reach if he or she moved by keeping the current velocity. Virtual goals also hold the expected time $t_{\mathrm{g}}$ until which the person would attain the goal. In other words, the position of the individual $p_{i}$ is projected into the future for a short time interval (proportional to the tracker cycle time) and denoted goal ahead time $\Delta g=$ $k \Delta t$. The location of the virtual goal $\mathbf{g}$ and the expected goal time $t_{\mathbf{g}}$ are defined as

$$
\mathbf{g}_{t}=\boldsymbol{x}_{t_{o}}+\boldsymbol{v}_{t_{o}}\left(t-t_{o}+\Delta g\right) \quad t_{\mathbf{g}}=t+\Delta g,
$$

where $t_{o}$ denotes the time when we last observed the person and $t$ the actual time. Note that the virtual goal is not fixed but moves along the last estimate of the velocity vector as time goes by. This behavior is needed as we do not know the duration of an occlusion event in advance and therefore we adjust the virtual goals on-the-fly.

Once $\mathbf{g}_{t}$ is estimated, the intended direction $\widehat{\boldsymbol{e}}_{t}$ and velocity $\widehat{\mathrm{v}}_{t}$ can be calculated using the offset between $\mathbf{x}_{t}$ and $\mathbf{g}_{t}$ and the expected goal time $t_{\mathbf{g}}$

$$
\widehat{\boldsymbol{e}}_{t}=\frac{\mathbf{g}_{t}-\boldsymbol{x}_{t}}{\left\|\mathbf{g}_{t}-\boldsymbol{x}_{t}\right\|} \quad \widehat{\mathrm{v}}_{t}=\frac{\left\|\mathbf{g}_{t}-\boldsymbol{x}_{t}\right\|}{t_{\mathbf{g}}-t} .
$$

For long term motion prediction the assumption of a virtual target destination can be generalized to goals that can either be learned by observing motion trajectories [7] or calculated from map representations [5], [8].

\section{B. Estimating Social Interactions}

While moving towards their virtual goal, the movements of the person are affected by the surrounding environment according to Eq. 2. The position $\boldsymbol{x}_{p}$ of all $p \in \mathcal{P}$ is provided by the tracking system, where each existing track is considered to be a separate individual. As for the static objects, we assume that no representation of the environment is known in advance and we estimate it using an occupancy grid framework. The grid is built by using the most recent laser observations (the last 30 in the experiments) and discarding points that are detected as people from our detection algorithm. When the occupancy probability of a cell is above a predefined threshold, it is consider to be occupied and inserted in the set of static obstacles $\mathcal{O}$. The center of this cell is then used as its position $\boldsymbol{x}_{o}$.

Once the surrounding people are known and the environment is learned, the interaction force exerted from $k \in \mathcal{P} \cup \mathcal{O}$ can be calculated following Eq. 4, where the normalized vector pointing from $k$ to $p_{i}$ is $\boldsymbol{n}_{i, k}=$ $\frac{\boldsymbol{x}_{i}-\boldsymbol{x}_{k}}{\left\|\boldsymbol{x}_{i}-\boldsymbol{x}_{k}\right\|}$. Since the laser range finder is sensing the surfaces of the obstacles, we assume they have no radius, i.e. $r_{k}=0$, and the sum of the radii $r_{i, k}$ is set to the radius of the individual $p_{i}$.

The grid is meant to be a short-term memory. In case of a mobile sensor, subsequent laser observations need to be registered into the common reference frame of the grid, either by using odometry, scan matching or mapbased localization.

\section{Estimating Physical Forces}

Physical forces according to Eq. 6 model the close contact interaction between two rigid bodies and express the principle that two different bodies cannot occupy the same space. The position of a person $\boldsymbol{x}_{p}$ and of a static object $\boldsymbol{x}_{o}$ and the direction of the force $\boldsymbol{n}_{i, k}$ are obtained in the same way as for the interaction forces described above. Physical forces are effective only when a physical contact is present (model by the function $g(\cdot)$ in Eq. 7 ).

The choice of having an occupancy grid instead of raw data as static obstacles has the advantage of constant force density. Using raw data, the density would vary over different parts of the environment, making the system behave differently. With an occupancy grid, it is possible to adjust and control the density of static obstacles by the cell size.

\section{EXPERIMENTS}

To experimentally evaluate the social force model and analyze its behavior, we integrate it within an MHTbased people tracker described in Arras et al. [4]. The Multi-Hypothesis Tracking (MHT) approach, based on the work of Reid [16] and Cox and Hingorani [17], hypothesizes about the state of the world by considering all statistically feasible assignments between measurements and tracks and all possible interpretations of measurements as false alarms or new track and tracks as matched, occluded or obsolete. A hypothesis $\Omega_{i}^{t}$ is one possible set of assignments and interpretations at time $t$. As the motion of an individual track is now influenced by other tracks in a hypothesis $\Omega_{i}^{t}$, motion predictions cannot be done on a per-track basis but must be done on a perhypothesis basis. The track tree proposed by Kurien [18] is a data structure that holds tracks from all hypothesis to exploit the fact that frequently, several hypothesis share the same tracks. This optimization technique has 


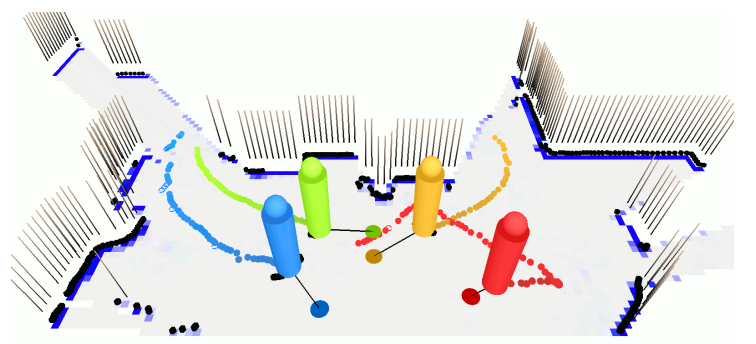

Fig. 5. Four of 135 tracks from the indoor experiment. The cylinders show the estimated positions of the pedestrians, the colored dots illustrate their past trajectories. The circles connected with lines are the virtual goals. The occupancy grid map is overlaid where darker blue cells indicate higher occupancy probability. Vertical lines visualize static objects.

to be abandoned with the social force model leading to increased memory requirements of our implementation.

Note that although we use the MHT as a tracking approach, our model is general and can be integrated in any existing tracking method.

We compare the tracking performance between the constant velocity motion model, the social force model and the social force model where only inter-human influences are considered. The data association error is used as a comparison measure, that is the number of identifier switches over the life cycle of a a tracked compared to ground truth. This value is indication of the tracking accuracy, especially of the ability to deal with occlusions.

We have collected two data sets: the first one in our laboratory (Fig. 5) and a second one in the city center of Freiburg (Fig. 1). The data have been recorded at $12 \mathrm{~Hz}$ using a fixed SICK laser scanner with an angular resolution of 0.5 degree mounted 0.8 meter above the floor. We use a pruning strategy that limits the maximum number of hypotheses of the MHT at every step to $N_{\text {Hyp }}$ (the multi-parent variant of the pruning algorithm proposed by Murty [19]). In order to show the evolution of the error as a function of the computational effort, $N_{H y p}$ is varied from 5 to 100 in the indoor experiment and from 50 to 1000 in the outdoor experiment.

To initialize the social force model, we assume that a general person has a radius of 0.2 meter and a mass of $80 \mathrm{~kg}$. The anisotropic blending factor of Eq. 4 is defined to be 0.5 , the goal ahead time is set to 60 tracking cycles and the relaxation time to 0.5 seconds. The parameters for the social forces exerted by obstacles are $a=100 \mathrm{~N}$ and $b=0.01 \mathrm{~m}$. For the social forces between persons we set $a=70 N$ and $b=0.4 \mathrm{~m}$. Physical forces from obstacles have a magnitude of $c=600 \mathrm{~N} / \mathrm{m}$ where the magnitude of the physical forces between persons is $c=250 \mathrm{~N} / \mathrm{m}$. These values have been determined experimentally from a set of calibration trials. The functions are plotted in Fig. 4.

\section{A. Indoor Environment}

The indoor data set from our laboratory consists of 38994 frames with a total number of 135 people entering

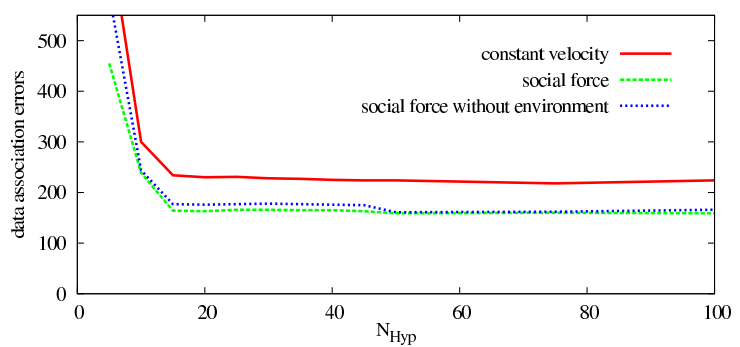

Fig. 6. Total number of data association errors as a function of $N_{H y p}$, the maximum number of hypotheses for the MHT algorithm to maintain. The solid red line shows the MHT tracker using the constant velocity motion model, the dashed green line the approach using the social force motion model and the dotted blue line the approach using inter-human influences only. The social force model makes about $30 \%$ fewer data association errors.

and leaving the sensor field of view. Both the detection and the data association have been labeled by hand to yield the ground truth.

The result shows a clear improvement of the MHT using the social force motion model over the regular approach with constant velocity motion model (Fig. 6). During occlusion events the tracker was able to avoid predictions into other people or walls leading to a more likely confirmation of tracks when they reappeared. Given the same number of hypotheses, the social force model makes around $30 \%$ fewer data association errors. Comparing the results of the two social force variants, the influence of the environment is smaller than initially expected.

The insight of this behavior is that better motion predictions lead to smaller innovations in the Kalman filter which translates to higher likelihoods as now the observations are better explained by the predictions. As the probability of a hypothesis directly depends on the likelihoods, the outcome is a more informed probability distribution over hypothesis. Consequently, the MHT tracker achieves similar performance at lower cost, or alternatively, higher efficiency at the same accuracy (see e.g. [4] for more details on the MHT theory).

\section{B. Outdoor Environment}

The second experiment has been carried out in the city center of Freiburg during a regular workday. The data set consists of 55475 frames during 25 minutes. 8600 frames with 150 persons have been labeled by hand, again to determine the ground truth detections and data associations. Occlusions are even more likely in such an scenario as more people move in a larger space. The resulting data association error is shown in Fig. 8.

The result shows an even more significant improvement by the social force model especially for smaller numbers of $N_{\text {Hyp }}$. Again, the contribution of the forces exerted by static obstacles is small, though higher than in the indoor case. For more than 800 hypotheses the social force model is still $16 \%$ better (644 versus 541 data association errors) than the constant velocity motion model. 

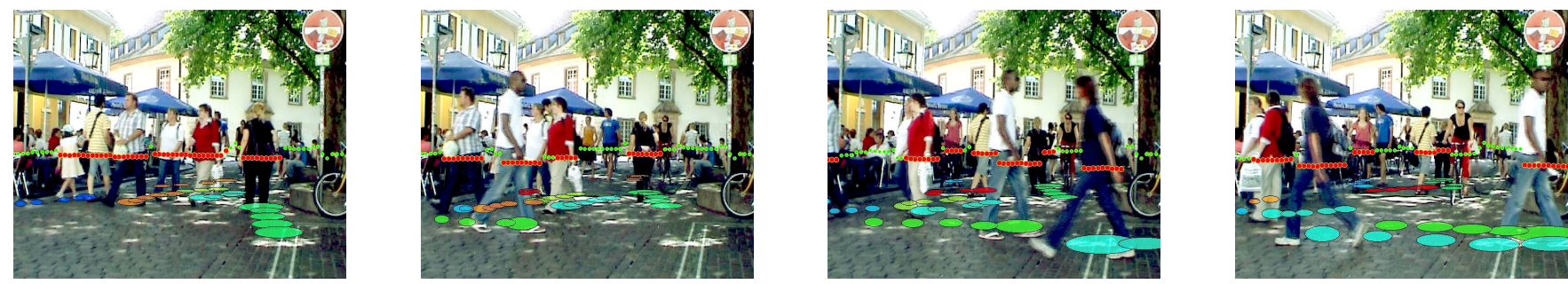

Fig. 7. Four images of the outdoor experiments carried out in the inner city of Freiburg. The information of the laser range finder and the tracking system were projected onto a recorded video sequence. The images from left to right show the tracking results at timesteps $t=335,353,365$ and 381. Laser range measurements are shown as small green dots (background) or small red dots (detected pedestrians). The traces of the observed pedestrians are drawn with colored ellipses.

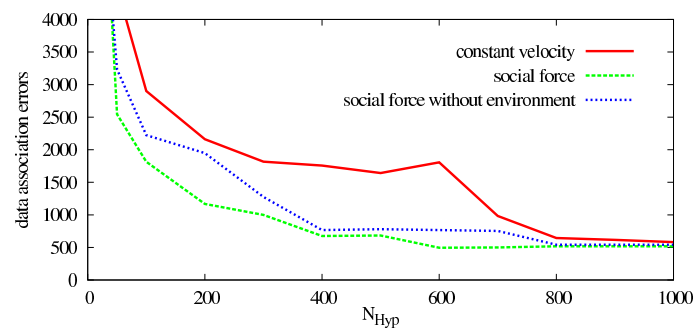

Fig. 8. Total number of data association errors as a function of $N_{\text {Hyp }}$. The solid red line shows the MHT tracker using the constant velocity motion model, the dashed green line the approach using the social force motion model and the dotted blue line the approach using inter-human influences only. The graph shows that the tracker requires fewer hypothesis to reach the same accuracy and, given more hypothesis, makes up to $50 \%$ fewer data association errors.

\section{Conclusions}

In this paper we presented a novel approach to human motion prediction based on social forces and environmental constraints. It overcomes the assumption typically made in related work of constant target velocity or acceleration. Our approach extends state-of-the-art by taking into account the influence of other people for human motion prediction. This is achieved with a single coherent model able to describe complex motion with a sound mathematical formulation. We showed how the social force model can be easily incorporated into a Kalman filter-based multi-hypothesis tracker.

The experiments, carried out in indoor and outdoor environments, demonstrate that our approach reduces the data association error by up to a factor of two. We found that the contribution of the interaction forces from the environment is small with respect to the forces from other people. This outcome is noteworthy and underlines the importance of considering other people in the prediction of human motion.

In future work, we will analyze friction and attractive forces, expressed within the social force model, to improve motion prediction for group tracking.

\section{ACKNOWLEDGMENT}

This work has been supported by the German Research Foundation (DFG) under contract number SFB/TR-8.

\section{REFERENCES}

[1] A. Fod, A. Howard, and M. Mataríc, "Laser-based people tracking," in Proc. of the Int. Conf. on Robotics $\&$ Automation (ICRA), 2002.
[2] D. Schulz, W. Burgard, D. Fox, and A. Cremers, "People tracking with a mobile robot using sample-based joint probabilistic data association filters," International Journal of Robotics Research (IJRR), vol. 22, no. 2, pp. 99-116, 2003.

[3] J. Cui, H. Zha, H. Zhao, and R. Shibasaki, "Tracking multiple people using laser and vision," in Proc. of the IEEE/RSJ Int. Conf. on Intelligent Robots and Systems (IROS), Alberta, Canada, 2005.

[4] K. O. Arras, S. Grzonka, M. Luber, and W. Burgard, "Efficient people tracking in laser range data using a multi-hypothesis leg-tracker with adaptive occlusion probabilities," in Proc. of the Int. Conf. on Robotics \& Automation (ICRA), 2008.

[5] A. Bruce and G. Gordon, "Better motion prediction for peopletracking," in Proc. of the Int. Conf. on Robotics 85 Automation (ICRA), Barcelona, Spain, 2004.

[6] L. Liao, D. Fox, J. Hightower, H. Kautz, and D. Schulz, "Voronoi tracking: Location estimation using sparse and noisy sensor data," in Proc. of the IEEE/RSJ Int. Conf. on Intelligent Robots and Systems (IROS), 2003.

[7] M. Bennewitz, W. Burgard, G. Cielniak, and S. Thrun, "Learning motion patterns of people for compliant robot motion," Int. Journal of Robotics Research (IJRR), vol. 24, 2005.

[8] M. Luber, G. D. Tipaldi, and K. O. Arras, "Place-dependent people tracking," in Proc. of the Int. Symposium of Robotics Research (ISRR), 2009.

[9] A. Schadschneider, W. Klingsch, H. Klüpfel, T. Kretz, C. Rogsch, and A. Seyfried, "Evacuation dynamics: Empirical results, modeling and applications," in Encyclopedia of Complexity and Systems Science, 2009, pp. 3142-3176.

[10] S. Hoogendoorn, "Gas kinetic modelling and simulation of pedestrian flows," in Proceedings of the Transportation Research Board, Washington, 2000.

[11] C. Burstedde, K. Klauck, A. Schadschneider, and J. Zittartz, "Simulation of pedestrian dynamics using a 2-dimensional cellular automaton," Physica A, vol. 295, pp. 507-525, 2001.

[12] S. Ali and M. Shah, "Floor fields for tracking in high density crowd scenes," in Proc. of the 10th European Conference on Computer Vision (ECCV). Springer-Verlag, 2008, pp. 1-14.

[13] D. Helbing, I. J. Farkás, P. Molnár, and T. Vicsek, "Simulation of pedestrian crowds in normal and evacuation situations," in Pedestrian and Evacuation Dynamics, M. Schreckenberg and S. D. Sharma, Eds. Springer, Berlin, Germany, 2002.

[14] S. Pellegrini, A. Ess, K. Schindler, and L. van Gool, "You'll never walk alone: modeling social behavior for multi-target tracking," in Int. Conf. on Computer Vision (ICCV), 2009.

[15] D. Helbing, I. Farkas, and T. Viscek, "Simulating dynamical features of escape panic," Nature, vol. 407, pp. 487-490, 2000.

[16] D. B. Reid, "An algorithm for tracking multiple targets," IEEE Transactions on Automatic Control, vol. 24, no. 6, 1979.

[17] I. J. Cox and S. L. Hingorani, "An efficient implementation of reid's multiple hypothesis tracking algorithm and its evaluation for the purpose of visual tracking," IEEE Trans. Pattern Anal. Mach. Intell. (PAMI), vol. 18, no. 2, pp. 138-150, 1996.

[18] T.Kurien, "Issues in the design of practical multitarget tracking algorithms," in Multitarget-Multisensor Tracking: Advanced Applications. Artech House, 1990, pp. 43-83.

[19] K. Murty, "An algorithm for ranking all the assignments in order of increasing cost," Operations Research, vol. 16, 1968. 